Oper Orthop Traumatol 2019.31:2

https://doi.org/10.1007/s00064-018-0583-3

(c) Springer Medizin Verlag $\mathrm{GmbH}$, ein Teil von Springer Nature 2018

CrossMark

\author{
A. B. Imhoff \\ Abt. f. Sportorthopädie, Klinikum rechts der Isar, München, Deutschland
}

\title{
Ligamentversorgung am Knie
}

Die Versorgung von einem multiligamentgeschädigten Kniegelenk hat sich durch die vielen neuen arthroskopisch geführten Versorgungsmöglichkeiten in den letzten Jahren massiv verändert. Während in der alten Nomenklatur mindestens 2 von 4 Hauptligamenten betroffen sein mussten, um von einer Multiligamentschädigung $\mathrm{zu}$ sprechen, sehen wir heute die Frühversorgung aller 4 Pfeiler adäquater. Da unter Experten ein eigentlicher Konsensus, wann welche Ligamente einzeitig oder mehrzeitig versorgt werden sollen, nach wie vor fehlt, war es uns ein Anliegen, ein Schwerpunktheft der Zeitschrift Operative Orthopädie und Traumatologie dieser Thematik zu widmen. Denn insbesondere die Verwendung von nichtresorbierbaren Fäden und Bändern wie FiberWire und FiberTape mit kleinen nichtresorbierbaren Ankersystemen machen eine Frühversorgung mit Naht bzw. Rekonstruktion und/oder Augmentation einfacher und sinnvoller, weil die zerstörten, teilweise zerfetzten, aber oft noch in wichtigen Struktureinheiten erhaltenen Ligamentteile für die Rekonstruktion verwendet werden können. So haben auch kürzliche, systematische Reviews demonstrieren können, dass sich mit einer frühen operativen Versorgung von multiligamentverletzten Kniegelenken im Vergleich zu konservativen bzw. nichtoperativen oder verzögerten Operationen eine verbesserte Funktion und ein besseres klinisches Outcome erreichen lässt.

In diesem Heft haben wir mehrere arthroskopische Rekonstruktionstechniken für die Refixation des hinteren Kreuzbandes, sei es an der tibialen oder femoralen Insertion, die Kombination mit internem Bracing oder schließlich die kombinierte Rekonstruktion des hinteren und des vorderen Kreuzbandes zusammengefasst. Auch ist wieder vermehrt das Augenmerk auf die Peripherie gerichtet, denn in Zusammenhang mit der Multiligamentverletzung muss auch oft anterolateral eine zusätzliche Stabilisierung extraartikulär als Anterolaterales Ligament (ALL) oder modifizierte Lemaire-Plastik in den Therapieplan integriert werden. Multiligamentverletzungen können auch die gleichzeitige Ruptur der Patellarsehne beinhalten, weshalb wir auch dieser Sehne ein Kapitel widmen und hier die modernen Augmentationsmethoden vorstellen wollen.

Es ist uns eine große Ehre, dieses Schwerpunktheft gestalten zu können und freuen uns auf jede Rückmeldung, alle Kommentare und Verbesserungsvorschläge aus unserer Leserschaft.

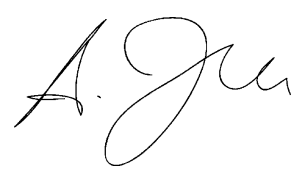

Univ.-Prof. Dr. A. B. Imhoff

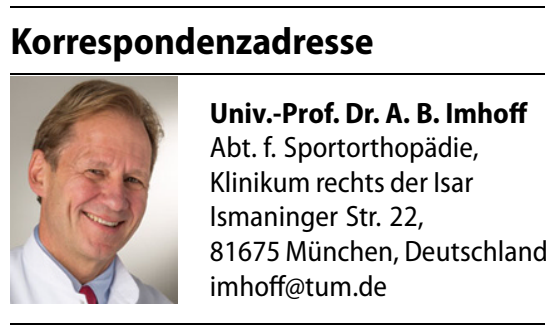

Interessenkonflikt. A.B. Imhoff gibt an, dass kein Interessenkonflikt besteht. 